Databases, Revenues, \& Repertory: The French Stage Online, 16801793 - Données, recettes \& réper toire: La scène en ligne (1680-1793)

\title{
Commentaire - Retour vers le futur
}

Dan Edelstein

Published on: Oct 07,2020

DOI: 10.21428/671d579e.2760cbcb

License: Creative Commons Attribution 4.0 International License (CC-BY 4.0). 
Quel type de savoir les projets en humanités numériques permettent-ils de construire ? Voici l'une des questions à l'origine des trois essais rassemblés dans la section «Interpréter les archives : données, visualisations, sons » du volume Données, recettes et répertoire. La scène en ligne (1680-1793) dirigé par Sylvaine Guyot et Jeffrey S. Ravel. Même si chaque article apporte une réponse différente à cette question, leurs divergences ne sont pas aussi marquées qu'il pourrait y paraître de prime abord.

En historien, Jeffrey_Ravel montre que le Projet des Registres de la Comédie-Française ( $\underline{R C F})$ s'inscrit dans la lignée d'une série d'entreprises antérieures de datafication, la première remontant au XVIII ${ }^{\mathrm{e}}$ siècle ${ }^{1}$. La numérisation des données n'en change pas fondamentalement le contenu : si la quantité d'informations augmente par ce biais de manière exponentielle, leur nature demeure relativement stable.

Jeffrey_Peters, quant à lui, considère que la visualisation des données engendre une quasi rupture épistémologique avec le type de savoir permis par l'imprimé 2 ; elle est, selon lui, « ouverture du nouveau et vers le nouveau ». Les interfaces utilisateurs créées par les humanités numériques reconfigurent nos interactions avec les objets culturels et transforment de ce fait notre expérience.

Pour Juliette Cherbuliez, l'archive numérique nous invite à explorer ce qui ne s'y trouve pas $\underline{3}$. Les lacunes s'accumulent aussi vite que les données et les humanités numériques posent davantage de questions qu'elles n'apportent de réponses. Dans le cas précis, elle s'interroge sur ce que les données sur la fréquentation de la ComédieFrançaise pourraient nous apprendre de l'environnement sonore qui était celui du théâtre.

Ces réactions au Projet $\underline{\mathrm{RCF}}$, et plus généralement aux humanités numériques, constituent des perspectives dans une certaine mesure distinctes les unes des autres. Pour schématiser, on trouve les positions suivantes : plus ça change, plus c'est la même chose, selon J. Ravel ; tout change, selon J. Peters ; et pour J. Cherbuliez, que sais-je ? Se pourrait-il toutefois que les humanités numériques engendrent ces trois réactions en même temps ? Loin d'être incompatibles, ne seraient-elles pas complémentaires ? C'est par l'affirmative que je voudrais, dans la suite de ces remarques, tenter de répondre à cette question.

L'une des forces du travail de J. Ravel est d'historiciser ce que nous aimons à considérer dans notre provincialisme temporel comme un développement récent. Si les 
tableurs n'existent que depuis les années 1980 et si la visualisation de données s'est rapidement développée au cours des vingt dernières années, les historiens nous rappellent que certaines des visualisations dont nous sommes les plus fiers ont une longue histoire ${ }^{4}$. Depuis les diagrammes circulaires de William Playfair (1801) et les cartes de flux utilisées par Charles-Joseph Minard pour la campagne de Russie napoléonienne (1869), jusqu'aux superbes graphiques sociologiques de W.E.B. Du Bois $\underline{5}$, la visualisation de données était déjà chose commune dans la culture de l’imprimé.

D'après ma lecture de l'article de J. Ravel, les entreprises plus anciennes pour traiter les données des registres de la Comédie-Française n’offraient pas de visualisations à proprement parler. Cependant, J. Ravel montre avec élégance que les tableaux proposés recelaient déjà une qualité proto-visuelle. Il cite un compte rendu critique de 1921 expliquant que les tableaux de données présents dans l'ouvrage d'Alexandre Joannidès, La Comédie-Française de 1680 à 1920, dessinent comme une " courbe devant les yeux ». Dans son analyse de ces tableaux, J. Ravel note que l'intérêt pour les pièces de Voltaire « se replie [tail off] de manière vertigineuse après 1850 » - la métaphore visuelle cristallisant ici ce qui apparaît avec évidence dans le tableau, qui en effet donne presque à voir un graphique. J. Ravel souligne lui-même que ces tableaux imprimés fonctionnent déjà comme des interfaces utilisateurs.

Ce travail important d'historicisation n'invalide pas l'hypothèse de J. Peters, à savoir que la nature « interfacielle » du Projet $\underline{\mathrm{RCF}}$ modifie en profondeur la manière dont nous concevons les phénomènes artistiques dont rendent compte les données. Il suggère simplement que ce changement s'est opéré plus anciennement : il n'a pas fallu attendre l'ère numérique pour voir apparaître de nouveaux rapports entre les chercheurs et les objets culturels qu'ils étudient.

Parmi les nombreuses propositions de J. Peters, je voudrais revenir en particulier sur l'idée que « les interfaces graphiques [sont] comme une forme de production et de réalisation plutôt qu'un mode de reconnaissance figé ». Cette idée met en lumière le statut épistémologiquement ambigu des données. Comme la plupart des sources de données, le Projet $\underline{\mathrm{RCF}}$ ne nous dit pas simplement les «faits »; ou plus exactement, les « faits » qu'il présente - les recettes quotidiennes en l'occurrence - ne donne pas un accès sans médiation à la réalité du théâtre. Faire parler les données nécessite « une forme de production et de réalisation ». Elles peuvent aider à formuler des hypothèses sur les raisons pour lesquelles certaines pièces eurent plus de succès que d'autres mais, pour tester ces hypothèses, il faut recourir à des sources extérieures. 
Les données en elles-mêmes ne fournissent pas de connaissances directes sur le théâtre.

Alors que J. Peters conclut son propos en interrogeant le statut ontologique de l'interface (de quelle nature est la réalité à laquelle elle se réfère ?), J. Cherbuliez se concentre sur la valeur épistémologique de l'archive. Que peut-elle et que ne peut-elle pas nous apprendre ? La série de questions que formule J. Cherbuliez laisse bien supposer sa réponse : les archives numériques ne nous fournissent pas de conclusions définitives mais elles suscitent de nouvelles interrogations. " Est-ce que les comédies vendent moins de place que les tragédies ? » et « Les vendredis sont-ils plus calmes que les mercredis ? » sont deux des questions que pose J. Cherbuliez avant de se demander comment l'archive numérique pourrait ou non nous aider à concevoir l'expérience acoustique d'un spectateur du XVIII' siècle.

Exemplaire d'une utilisation possible de l'archive, l'article de J. Cherbuliez est particulièrement éclairant du fait des étapes méthodologiques par lesquelles elle passe. Ce n'est pas ce qu'il y a dans l'archive qui l'amène à s'interroger sur les sons, mais c'est l'action de la parcourir des yeux qui en fait apparaître les différents silences. Ces silences peuvent être des trous dans les archives, ce que S. Guyot et J. Ravel décrivent dans l'introduction du volume comme des « lacunes dans les sources [historiques] elles-mêmes ». Il est d'autant plus facile d'identifier ce qui fait défaut que nous sommes mis en présence d'un grand nombre de données. Un autre type de silence résonne lorsque l'on ne voit pas - ou lorsque l'on n'entend pas - ce que l'on s'attendait à trouver, selon un décalage qui permet de « repérer des erreurs potentielles dans les données », écrit J. Cherbuliez. Dans certains cas, ce type de silence n'est qu'un écho du premier, mais pas toujours. Lanomalie peut être mystérieuse et inciter le chercheur à se lancer dans la quête de réponses. Enfin, les données existantes peuvent mettre en évidence la nature des données qui manquent. C'est ce qui arrive lorsque J. Cherbuliez, alors qu'elle examine les recettes quotidiennes, finit par s'interroger sur l'environnement sonore du théâtre.

Chacun à leur manière, ces trois articles soulignent comment la datafication et la quantification en sciences humaines ne rapprochent pas nécessairement leurs méthodes de celles des sciences sociales. La recherche en humanités numériques ne conduit pas nécessairement à privilégier les statistiques; chiffres et pourcentages servent souvent de point de départ à des enquêtes scientifiques, et ils ne produisent aucune certitude. Comme nous le rappelle J. Ravel, une grande partie de ces 
recherches n'ont pas attendu l'ère numérique pour débuter. Tout ce qui brille n'est pas neuf.

\section{Footnotes}

1. Jeffrey S. Ravel, « La Comédie-Française par les chiffres, 1752-2020», dans le présent volume. $\leftrightarrows$

2. Jeffrey Peters, «Voir le littéraire : la visualisation de données et le Projet des

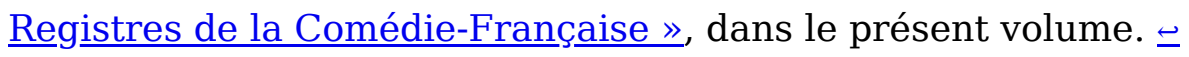

3. Juliette Cherbuliez, «Les sons du théâtre : publics, acoustique, déclamation », dans le présent volume. $\uplus$

4. Voir en particulier Daniel Rosenberg et Anthony Grafton, Cartographies of Time. A Visual History of the Timeline, New York, Princeton Architectural Press, 2010. Plus récemment, voir Paolo Ciuccarelli et Tommaso Elli, « Beyond Visualization », dans Howard Hotson et Thomas Wallnig (dir.), Reassemling the Republic of Letters in the Digital Age. Standards, Systems, Scholarship, Göttingen, Göttingen UP, 2019, https://doi.org/10.17875/gup2019-1146.

5. Voir Whitney Battle-Baptiste et Britt Earl (dir.), Du Bois's Data Portraits. Visualizing Black America, Amherst, The W.E.B. Du Bois Center at the University of Massachussetts Amherst et New York, Princeton Architectural Press, 2018. 